\title{
Stability Analysis of Robust Multiple Model Adaptive Control
}

\author{
Vahid Hassani ${ }^{*}$ João Pedro Hespanha ${ }^{* *}$ Michael Athans ${ }^{*, * * *}$ \\ António M. Pascoal* \\ * Institute for Systems and Robotics (ISR), Instituto Superior Técnico (IST), \\ Lisbon, Portugal. Tel: (+351) 21841 8054, Fax: (+351) 218418291 \\ \{vahid, athans, antonio\}@isr.ist.utl.pt \\ ** Dept. of Electrical and Computer Eng., Univ. of California, Santa Barbara, \\ CA 93106-9560, USA, hespanha@ece.ucsb.edu. \\ *** Michael Athans is also Professor of EECS (emeritus), M.I.T., USA.
}

\begin{abstract}
The Robust Multiple Model Adaptive Control (RMMAC) methodology was first introduced in Fekri et al. [2006] for open-loop stable plants with parametric uncertainty and unmodeled dynamics subjected to external disturbances and measurement noise. This paper addresses the stability of RMMAC systems. We show, using concepts and analysis tools that borrow from Supervisory Control, that all closed-loop signals in a RMMAC system are bounded. It is further shown that robust performance is recovered in steady state.
\end{abstract}

Keywords: Robust Adaptive Control; Multiple Model Control; Supervisory Control.

\section{INTRODUCTION}

Adaptive control is often required to control plants with large parametric uncertainty for which a single controller cannot yield adequate robust stability and performance. Many approaches to the problem of adaptive control have been considered in the literature. In particular, Multiple Model Adaptive Control (MMAC) algorithms have received widespread attention; see for example Fekri et al. [2006, 2007], Lainiotis [1976], Schiller and Maybeck [1997], Safonov and Tsao [1997], Anderson et al. [2000], Hespanha et al. [2001, 2003], Hespanha [2001], and the references therein. The use of multiple models for estimation and control is by no means new. In the 1960s and 1970s, several authors including Lainiotis [1976] studied the problems of Multiple Model Adaptive Estimation and Control, referred to as MMAE and MMAC, respectively. Many other versions of adaptive control using multiple models have been reported in the last decade. Numerous results based on these methods can be found for example in Fekri et al. [2006, 2007], Schiller and Maybeck [1997], Safonov and Tsao [1997], Hespanha et al. [2001], and Hespanha et al. [2003]. Common to all these methods is the use of information obtained online to decide on appropriate control actions. The adaptive architectures proposed consist of a finite set of candidate controllers and an identification module. At least one of the candidate controllers is assumed to meet desired closed-loop stability and performance requirements for the plant in the loop. It is the role of the identification module to

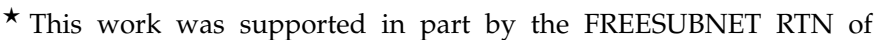
the CEC, projects Co3-AUVs (EU FP7 under grant agreement No. 231387), NSF Grant ECCS-0725485 and DENO / FCT-PT (PTDC/EEAACR/67020/2006), and the FCT-ISR/IST plurianual funding program (through the POS $\mathrm{C}$ initiative in cooperation with FEDER). The first author benefited from grant SFRH/BD/45775/2008 of the Foundation for Science and Technology (FCT), Portugal.
}

select a controller whose performance is "better" than that obtained with the other controllers in the candidate set of controllers. Except for unfalsified control (exposed in Safonov and Tsao [1997]), which is not a model-based approach and utilizes measured data to falsify (and assess the performance of) controllers in and out of the loop in real time based on a cost function, the other techniques use a model-based approach. Furthermore, their identification modules compute a set of performance signals which are suitably defined indexes based on the output estimation errors with respect to a set of candidate reference models.

A promising multi-controller approach is based on the socalled robust multiple model adaptive control (RMMAC) methodology that provides guidelines for designing both the bank of candidate controllers (using mixed- $\mu$ synthesis tools) and the identification module, see Fekri et al. [2006, 2007]. In the RMMAC methodology, the parameter uncertainty set is divided into smaller regions and local robust controllers $K_{i}$, one for each region, are designed using the $\mu$-synthesis method described for example in Balas [2009]. The number of regions and consequently of robust local controllers is not selected by trial and error, but is rather a direct consequence of the design specifications for the adaptive system. In this approach, the designer specifies that an appropriately defined performance index should be equal or greater than a certain percentage, say $\mathrm{X} \%$, of the best possible performance achievable if the uncertain parameters were known exactly. In order to select the correct controller, the RMMAC embodies in its structure a bank of Kalman filters (KFs), the number of which is equal to that of the controllers.

Each KF is designed for a plant model corresponding to a selected value of the unknown parameter. The choice of the design parameters and consequently of the design models is the outcome of a clearly defined methodology. The residuals of all the KFs are analyzed in a block called 
the Posterior Probability Evaluator (PPE) that assigns the posterior probability $p_{i}$ to the $i^{\text {th }}$ plant model. These posterior probabilities are used to select the appropriate local controller (the one associated with the highest posterior probability). Proper design of each KF in the RMMAC architecture is absolutely essential in order to satisfy the theoretical assumptions which will imply that the Posterior Probability Evaluator (PPE) will yield correct model identification. Fekri et al. [2006, 2007] used the Baram Proximity Index (BPI) to design the nominal KFs in the RMMAC to ensure that if the underlying theoretical assumptions are satisfied then the correct controller will be selected.

Notwithstanding the superior performance of the RMMAC architecture shown with the help of simulations in Rosa et al. [2007a,b], no stability analysis is available in the literature. The main contribution of this paper is the proof that with a small change in the structure of the original RMMAC, and without any extra assumptions, all the resulting closed-loop internal signals are bounded. It is further shown that robust performance is recovered in steady state when the underlying plant is time-invariant. The above results are important steps towards the development of a solid theoretical framework for the design of robust adaptive control systems for open-loop stable plants with parametric uncertainty, subjected to external disturbances and measurement noise. The methodology adopted seeks inspiration from concepts and mathematical tools developed in the scope of supervisory control theory (see Hespanha et al. [2001, 2003] and the references therein).

The structure of the paper is as follows. Section 2 reviews the structure of the RMMAC and describes its new switching version. The stability analysis of the resulting architecture for robust adaptive control is presented in Section 3. Finally, the conclusions and suggestions for future research are summarized in Section 4.

\section{ROBUST ADAPTIVE CONTROL USING MULTIPLE CONTROLLERS}

The robust multiple model adaptive control (RMMAC) methodology was introduced to deal explicitly with large parametric uncertainty and unmodelled dynamics. See Fekri et al. [2006, 2007], Hassani et al. [2010b] and the references therein for an explanation of the basic architecture that involves a bank of controllers and an identification module. In what follows, and adopting the notation introduced in the above references, we assume that the plant model $G$ of interest is subjected to parameter uncertainty $\theta$, that is, $G=G(\theta)$ where $\theta$ lives in some compact finite dimensional set. We consider discretetime multiple-input-multiple-output (MIMO) linear timeinvariant (LTI) plant models $G$ of the form

$$
\begin{aligned}
x(t+1) & =A_{\theta} x(t)+B_{\theta} u(t)+G_{\theta} w(t), \\
y(t) & =C_{\theta} x(t)+v(t),
\end{aligned}
$$

where $x(t) \in \mathbb{R}^{n}$ denotes the state of the system, $u(t) \in \mathbb{R}^{m}$ its control input, $y(t) \in \mathbb{R}^{q}$ its measured noisy output, $w(t) \in \mathbb{R}^{r}$ an input plant disturbance that cannot be measured, and $v(t) \in \mathbb{R}^{q}$ is the measurement noise. The vectors $w(t)$ and $v(t)$ are zero-mean, mutually independent white Gaussian sequences, with covariances $\operatorname{cov}[w(t) ; w(\tau)]=Q \delta_{t \tau}$ and $\operatorname{cov}[v(t) ; v(\tau)]=R \delta_{t \tau}$, respectively. The initial condition $x(0)$ of $(1 \mathrm{a})$ is a Gaussian random vector with mean and covariance given by $E\{x(0)\}=0$ and $E\left\{x(0) x^{T}(0)\right\}=\Sigma(0)$. The matrices $A_{\theta}, B_{\theta}, G_{\theta}$, and $C_{\theta}$ contain an unknown constant parameter indexed by vector $\theta \in \Omega \subset \mathbb{R}^{l}$, where $\Omega$ is a convex compact set. In the original RMMAC architecture of Fekri et al. [2006, 2007], posterior probabilities are computed to weigh the local control actions from a bank of controllers. Similarly, a switching version of the RMMAC is reported in Rosa et al. [2007a,b], where posterior probabilities are used to select and switch one controller from the bank of controllers. Figs. 1 (together with identification module shown in Fig. 2) show the architecture of the switching version of the RMMAC that is at the root of the main results in the paper. In this architecture, the identification module (that relies on a posterior probability evaluator) computes the highest posterior probability $p_{j} ; j \in\{1,2, \ldots N\}$ and switches the controller $K_{j}$ associated with that posterior probability, after which it dwells on that selection for a predefined time, called dwell-time. The RMMAC depicted in Fig. 1 has a modular structure. The overall controller consists of two main components: a bank of local controllers and an identification unit. The identification unit of the switching version of the RMMAC is depicted in Fig. 2; it is made up of 1) a bank of observers, 2) a posterior probability evaluator, and 3) the controller selection unit with dwelltime.

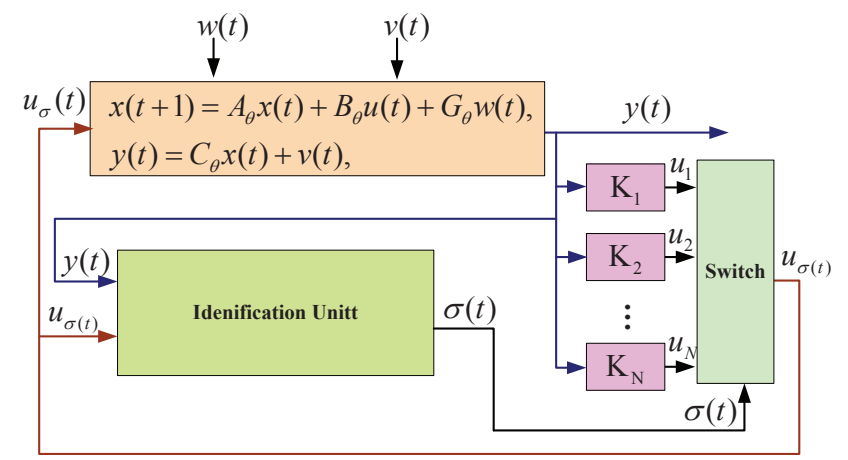

Fig. 1. A switched version of the RMMAC architecture in Fekri et al. [2006].

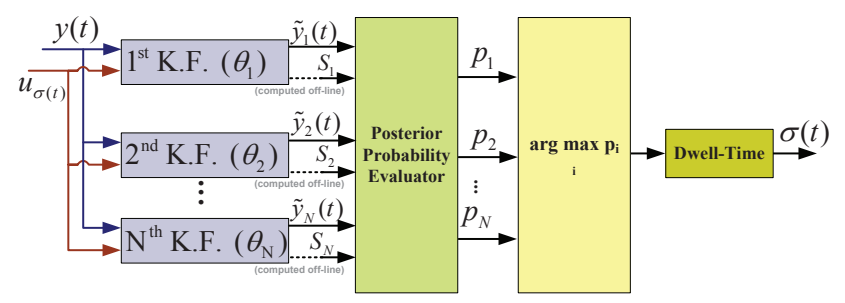

Fig. 2. Identification unit in the switched version of the RMMAC architecture.

The posterior probabilities are computed on-line by the posterior probability evaluator (PPE) using the recursive formula (see Sims et al. [1969] for more details)

$$
p_{i}(t+1)=\frac{\beta_{\theta_{i}}^{-1} e^{-m_{\theta_{i}}(t+1)}}{\sum_{j=1}^{N} p_{j}(t) \beta_{\theta_{j}}^{-1} e^{-m_{\theta_{j}}(t+1)}} p_{i}(t)
$$

where $p_{i}(0)$ are the prior model probabilities and $m_{\theta_{i}}(t)$ and $\beta_{\theta_{i}}$ are defined as 


$$
\begin{aligned}
m_{\theta_{i}}(t) & :=\frac{1}{2}\left[\hat{y}_{\theta_{i}}(t)-y(t)\right]^{T} \Sigma_{\theta_{i}}^{-1}\left[\hat{y}_{\theta_{i}}(t)-y(t)\right], \\
\beta_{\theta_{i}} & :=(2 \pi)^{\frac{q}{2}} \sqrt{\left|\Sigma_{\theta_{i}}\right|}
\end{aligned}
$$

with $q$ denoting the dimension of the measurement vector $y(t)$ and $\Sigma_{\theta_{i}}$ the covariance matrix of the residuals of the $i^{\text {th }} \mathrm{KF}$.

A slightly modified version of this identification unit which will be used and referred throughout this article, is depicted in Fig. 3. The only difference between Figs. 2 and 3 is the use of the monitoring signals (to be defined shortly) instead of the posterior probabilities. As mentioned before in the RMMAC, the selection of the local controller is done by comparing the computed posterior probabilities (see Fig. 2) while in Fig. 3 the controller selection is done by examining the monitoring signals. Later in this section, in Lemma 1, we will prove that Figs. 2 and 3 are equivalent. For our purposes, the RMMAC structure with identification unit shown in Fig. 3 is easier to deal with and will be adopted in the paper.

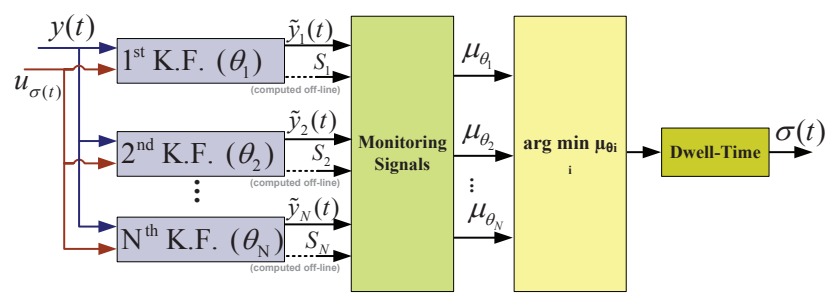

Fig. 3. Structure of the Identification unit with the Use of Monitoring Signals and Dwell-Time.

Corresponding to each of the sub-regions of the parametric uncertainty set in the RMMAC, a selected value of the unknown parameter is used to design a steady state Kalman filter. ${ }^{1}$ The output estimation errors and error covariances of all the Kalman filters are used to compute a performance signal that can be viewed as a gaussian maximum likelihood ratio. This signal is called a "monitoring signal" $2 \mu_{i}(t)$, and is defined as

$$
\mu_{\theta_{i}}(t):=\frac{1}{t} \sum_{k=1}^{t} m_{\theta_{i}}(k)+\ln \beta_{\theta_{i}},
$$

where $m_{\theta_{i}}(t)$ and $\beta_{\theta_{i}}$ are given in (3).

The monitoring signals are then used to select one of the local controllers (the one associated with Kalman filter with the smallest monitoring signal). The following lemma applies.

Lemma 1. Assume $p_{i}(0)=p_{j}(0)$, for all $i \& j \in\{1, \ldots, N\}$. Then,

$$
\underset{i=1, \ldots, N}{\operatorname{argmax}}\left\{p_{i}(t)\right\}=\underset{j=1, \ldots, N}{\operatorname{argmin}}\left\{\mu_{\theta_{j}}(t)\right\}, \quad t=1,2, \ldots
$$

A proof is available in Hassani et al. [2010a]. In what follows, the different modules of the modified RMMAC controller are introduced in detail.

\footnotetext{
1 For simplicity of analysis we assume that corresponding to each subinterval, only one $\mathrm{KF}$ is designed. However the number of observers can be larger than the number of controllers (see RMMAC/XI in Fekri et al. [2007]).

2 The term "monitoring signal" is borrowed from the work developed in Hespanha et al. [2001], Hespanha [2001]
}

\subsection{Bank of controllers}

In the RMMAC, the parametric uncertainty set, $\Omega$, is partitioned into $N$ smaller sub-regions $\Omega_{i}$ (compact and convex) and for each sub-regions a local controller is designed in such a way that it provides robust-stability and robust-performance for all the plants with possible parameter values in the corresponding sub-region. Moreover, in the RMMAC methodology, a systematic approach is presented to design the local controllers, see Fekri et al. [2007]. Here we assume that $N$ controllers $K_{i}$ with realizations

$$
\begin{aligned}
x_{i}^{c}(t+1) & =A_{i}^{c} x_{i}^{c}(t)+B_{i}^{c} y(t) \\
u(t) & =C_{i}^{c} x_{i}^{c}(t), \quad i \in\{1,2, \ldots, N\}
\end{aligned}
$$

have been designed. At each sampling instant the identification unit provides the signal $\sigma(t)$ and a local controller from the bank of controllers is selected and inserted in the loop. Stated mathematically,

$$
\begin{aligned}
x_{c}(t+1) & =A_{\sigma(t)}^{c} x_{c}(t)+B_{\sigma(t)}^{c} y(t) \\
u(t) & =C_{\sigma(t)}^{c} x_{c}(t) .
\end{aligned}
$$

\subsection{Identification Unit}

The identification unit decides which controller should be switched in the feedback loop and when this should take place. The monitoring signal generator unit associates a monitoring signal to each KF (from the bank of observers) based on the output estimation errors (and their covariance). Subsequently, the monitoring signals are assessed and a "dwell-time switching logic" produces the switching signal $\sigma(t)$ in Fig. 2. In fact, the dwell-time switching logic assigns $\sigma(t)=\operatorname{argmin}\left\{\mu_{\theta_{j}}(t)\right\}$, where $j=1, \ldots, N$, but "dwells" on this particular choice for at least a prespecified amount of time $\tau_{D}$ called the dwell-time to be explained in detail later.

In what follows, we review the main issues involved in the design and selection of the modules in the identification unit.

Bank of Estimators Corresponding to each sub-region in the parametric uncertainty set, a selected value of the unknown parameter is used to design a steady state Kalman filter of the form

$$
\begin{aligned}
\hat{x}_{\theta_{i}}(t+1) & =\left(A_{\theta_{i}}-A_{\theta_{i}} H_{\theta_{i}} C_{\theta_{i}}\right) \hat{x}_{\theta_{i}}(t)+B_{\theta_{i}} u(t)+A_{\theta_{1}} H_{\theta_{1}} y(t) \\
\hat{y}_{\theta_{i}}(t) & =C_{\theta_{i}} \hat{x}_{\theta_{i}}(t), \\
\tilde{y}_{\theta_{i}}(t) & =C_{\theta_{i}} \hat{x}_{\theta_{i}}(t)-y(t), \quad i \in\{1,2, \ldots, N\}
\end{aligned}
$$

where $H_{\theta_{i}}$ is the Kalman filter gain. The bank of estimators aims to provide a good estimation of the output $y(t)$. In fact, when the unknown parameter is within a particular subinterval, then the corresponding output estimate should be close to the process output and therefore the corresponding residual should be small. The reader is referred to Hassani et al. [2009] where a systematic algorithm is provided to decide on the number of observers and the selection of the nominal values to design the estimators that will guarantee a predefined level of performance, as evaluated by an index defined based on the estimation error.

Each KF is designed based on a selected value of the unknown parameter. In the standard RMMAC, the residuals 
of all the KFs are analyzed through a dynamic hypothesis testing block to compute the aposterior probability. These posterior probabilities are used to select the appropriate local controller (the one associated with the highest posterior probability).

Proper design of each KF in the RMMAC architecture is absolutely essential in order to satisfy the theoretical assumptions (see Fekri et al. [2006]) which will imply that the Posterior Probability Evaluator (PPE) will yield correct model identification. Baram and Sandell [1978] analyzed the convergence of the conditional probabilities $p_{i}$ and showed that the one corresponding to the KF designed for the "nearest" to the actual system (in a stochastic norm sense) converges to 1 , while the others tend to 0 . We refer to this norm as the Baram Proximity Index (BPI ${ }^{3}$ given by

$$
\Gamma_{\theta_{i}}^{\theta_{\star}} \equiv+\frac{q}{2} \ln (2 \pi)+\frac{1}{2} \ln \left(\left|\Sigma_{\theta_{i}}\right|\right)+\frac{1}{2} \operatorname{tr}\left(\Sigma_{\theta_{i}}^{-1} \Sigma_{\theta_{i}}^{\theta_{\star}}\right),
$$

where $\theta_{\star}$ is the true value of the unknown parameter, the $\theta_{i} \mathrm{~s}$ are the nominal values of the parameter used in design of the KFs, $\Sigma_{\theta_{i}}$ is the covariance of the residuals in the $i^{t h} \mathrm{KF}, \Sigma_{\theta_{i}}^{\theta_{\star}}$ is the covariance of the residuals when the system is running with the true parameter $\theta_{\star}$ but the KF designed based on $\theta_{i}$ is used in the estimation, and $q$ is the dimension of the measurement.

Baram and Sandell [1978] proved that in the multiple model approach the KF whose BPI, $\Gamma_{\theta_{i}}^{\theta_{\star}}$, is smaller than the others will be selected.

Fekri et al. [2006, 2007] used the BPI to design the nominal KFs in the RMMAC. The key idea exploited, illustrated for three models in Fig. 4 for a scalar parameter, is to use an iterative algorithm to calculate the nominal values $\theta_{1}$, $\theta_{2}$, and $\theta_{3}$ so that the BPIs are equal at the boundary of adjacent $\Omega_{i}$ 's. In this manner, the fundamental probability convergence result is $\theta \in \Omega_{i} \Rightarrow p_{i}(t) \rightarrow 1$ almost surely. This method becomes more complicated for two or more uncertain parameters (see Fekri et al. [2006]).

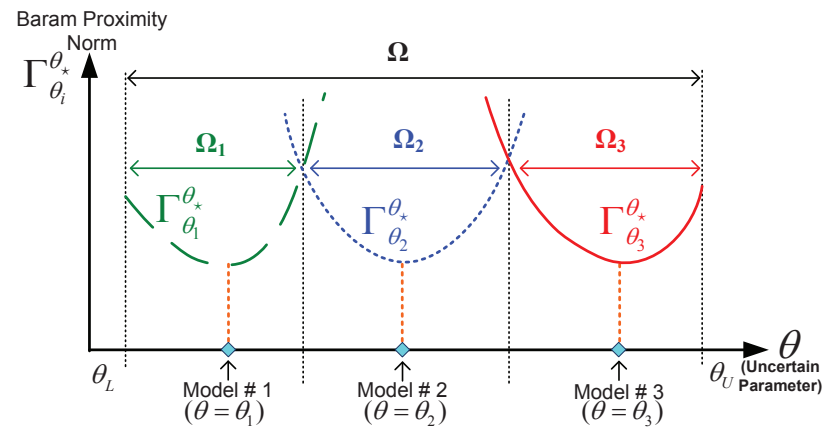

Fig. 4. Optimizing the KF nominal design points using the BPIs so that they are equal at the boundaries of adjacent models.

As a matter of fact, when the Kalman filters are designed in this manner, for every $\theta \in \Omega$, the Kalman filter associated with $\Omega_{i}$ where $\theta \in \Omega_{i}$, will exhibit a less value of BPI among other KFs. In other words, for every $\theta \in \Omega$ there exist a $\mu_{\theta_{i}}(t)$ whose value is bounded and smaller than the

\footnotetext{
3 Baram and Sandell [1978] developed a pseudo norm based on the BPI. With an obvious abuse of notation, throughout this paper, we refer to the BPI as a norm.
}

others. Clearly, this bound depends on the signals which drive the plant and the Kalman filters, i.e. $w(t), v(t)$, and $u(t)$. Formally, the following property is satisfied when the KFs are designed using the BPI approach:

$$
\begin{gathered}
\exists C_{0}, C_{w}, C_{v}, C_{\epsilon}, \epsilon>0 \text { such that } \forall \theta_{i} \in \Theta \text { and } \forall t>0 \\
\begin{array}{r}
\frac{1}{2}\left\|\tilde{y}_{\theta_{i}}^{T}(\tau) \Sigma_{\theta_{i}}^{-1} \tilde{y}_{\theta_{i}}(\tau)\right\|_{(0, t)} \leqslant C_{0}+C_{w}\left\|w^{T}(\tau) w(\tau)\right\|_{(0, t)} \\
+C_{v}\left\|v^{T}(\tau) v(\tau)\right\|_{(0, t)}+\epsilon C_{\epsilon}\left\|u^{T}(\tau) u(\tau)\right\|_{(0, t)} .
\end{array}
\end{gathered}
$$

where $\|$.$\| is defined as$

$$
\|\vartheta(s)\|_{(d, h)}=\sum_{\tau=d+1}^{h} \vartheta(\tau) .
$$

The monitoring signals introduced in (4) approach the BPI introduced in (9) as $t \rightarrow \infty$.

As mentioned before, in the RMMAC the design of the KFs starts only after the number of the local controllers is selected and the uncertainty set is split into smallers sets. By using the BPI tool in the process of designing the KFs, it is assured that for every $\theta \in \Omega_{i}$, the $i^{\text {th }} \mathrm{KF}$ will have a minimum BPI among all the KFs. This means that as $t \rightarrow \infty$, the $\mu_{\theta_{i}}(t)$ will have the smallest value among all the monitoring signals. Consequently, for every $\theta \in \Omega_{i}$, the controller $K_{i}(s)$ will be switched in the feedback loop as $t \rightarrow \infty$, which will yield robust stability and performance at steady state.

Selection Module At the heart of the adaptive control architecture of in Fig. 1 is the identification module, see Fig. 2. This module assesses the output error from the bank of Kalman filters at each sampling and evaluates, in real time, which estimator yields the best estimate of the process output (from which it follows the model that best matches the actual process). In order to do so, for each estimator a performance signal is computed from the output estimation error and error covariance (provided by each Kalman filter). Fig. 5 describes graphically the dwell-time switching logic utilized here, motivated by Hespanha [2001].

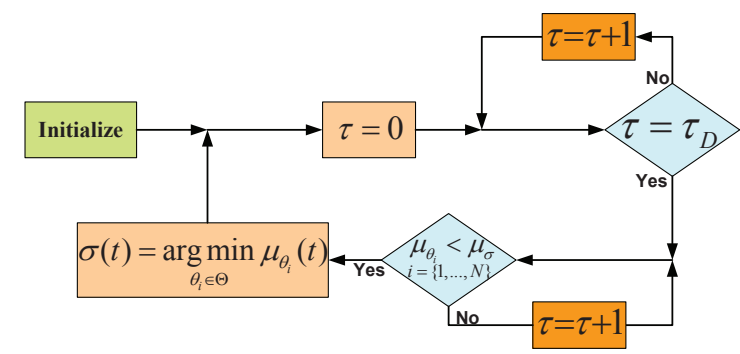

Fig. 5. The dwell-time switching logic.

Let assume that at time $t$ the controller $K_{i}$ is inserted in the feedback loop. Then, the dwell-time switching logic will keep the controller $K_{i}$ in the loop for the next successive $\tau_{D}$ sampling times, even if another estimator shows better performance meanwhile. This may cause connection of a wrong controller in the feedback loop. The following theorem ensures that using the so called "dwell-time switching logic" and the monitoring signals defined in (4), only for at most $(N+1) \tau_{D}$ sampling times, a wrong controller may be selected. 
Theorem 2. Let $\Theta$ denote the finite set of $N$ nominal values of the parameter vector $\theta$ selected for the design of the $N$ Kalman filters in the bank of estimators. For every $t \geqslant 0$ there exists a "virtual" switching signal $\tilde{\sigma}_{t}(\tau), 0 \leqslant \tau \leqslant t$ that differs from the real switching signal $\sigma(\tau)$ at most for $(N+1) \tau_{D}$ sampling times, and such that

$$
\left\|\mu_{\tilde{\sigma}_{t}(\tau)}(\tau)\right\|_{(0, t)} \leqslant N\left\|\mu_{\theta_{j}}(\tau)\right\|_{(0, t),} \quad \forall \theta_{j} \in \Theta .
$$

A proof is available in Hassani et al. [2010a]. At this stage, let us take the discussion a stage further and highlight the importance of (12). This property in supervisory control is called small error property and ensures that given a fixed dwell-time, the selection module always chooses the monitoring signal with minimum value, except for a finite predefined number of sampling times. The virtual switching signal in theorem 2 is the same as the real switching signal except for at most $(N+1) \tau_{D}$ sampling times.

\section{CLOSED-LOOP STABILITY ANALYSIS}

Assume for the time being that there is no dwell-time switching and that at each sampling time the controller $K_{i}$ is in the loop, as a consequence of the fact that $\theta_{i}=\operatorname{argmin}\left\{\mu_{\theta_{j}}(t)\right\}$. In this case, if we view the closed$j=1, \ldots, N$

loop switched system as the feedback interconnection of the process and an "injected system" (to be defined later), it can be easily verified that the latter is asymptotically stable. The injected system can be regarded as a dynamic system whose input and output are the output estimation error provided by the KF associated with the controller in the loop and the control action $u(t)$, respectively. In what follows, the dynamic of the injected system is derived. For simplicity, let us assume that we have only two estimators,

$$
\begin{aligned}
& {\left[\begin{array}{l}
\hat{x}_{\theta_{1}}(t+1) \\
\hat{x}_{\theta_{2}}(t+1)
\end{array}\right]=} \\
& {\left[\begin{array}{cc}
A_{E_{1}} & 0 \\
0 & A_{E_{2}}
\end{array}\right]\left[\begin{array}{l}
\hat{x}_{\theta_{1}}(t) \\
\hat{x}_{\theta_{2}}(t)
\end{array}\right]+\left[\begin{array}{l}
A_{\theta_{1}} H_{\theta_{1}} \\
A_{\theta_{2}} H_{\theta_{2}}
\end{array}\right] y(t)+\left[\begin{array}{l}
B_{\theta_{1}} \\
B_{\theta_{2}}
\end{array}\right] u(t)} \\
& {\left[\begin{array}{c}
\tilde{y}_{\theta_{1}}(t) \\
\tilde{y}_{\theta_{2}}(t)
\end{array}\right]=\left[\begin{array}{cc}
C_{\theta_{1}} & 0 \\
0 & C_{\theta_{2}}
\end{array}\right]\left[\begin{array}{l}
\hat{x}_{\theta_{1}}(t) \\
\hat{x}_{\theta_{2}}(t)
\end{array}\right]+\left[\begin{array}{l}
-I \\
-I
\end{array}\right] y(t)}
\end{aligned}
$$

where $A_{E_{i}}=A_{\theta_{i}}-A_{\theta_{i}} H_{\theta_{i}} C_{\theta_{i}}$, and two controllers, $K_{i}$,

$$
\begin{aligned}
x_{i}^{c}(t+1) & =A_{i}^{c} x_{i}^{c}(t)+B_{i}^{c} y(t) \\
u(t) & =C_{i}^{c} x_{i}^{c}(t),
\end{aligned}
$$

where $i=1,2$, are designed. Let us assume that at time $t$, we have $\mu_{\theta_{1}}<\mu_{\theta_{2}}$ which means that controller $K_{1}$ should be in the loop. Using the fact that $y(t)=\hat{y}_{\theta_{1}}-\tilde{y}_{\theta_{1}}$ and $u(t)=C_{1}^{c} x_{1}^{c}$, yields

$$
\begin{aligned}
& {\left[\begin{array}{c}
\hat{x}_{\theta_{1}}(t+1) \\
\hat{x}_{\theta_{2}}(t+1) \\
x_{1}^{c}(t+1)
\end{array}\right]=} \\
& {\left[\begin{array}{ccc}
A_{\theta_{1}} & 0 & B_{\theta_{1}} C_{1}^{c} \\
A_{\theta_{2}} H_{\theta_{2}} C_{\theta_{1}} & A_{E_{2}} & B_{\theta_{2}} C_{1}^{c} \\
B_{i}^{c} C_{\theta_{1}} & 0 & A_{1}^{c}
\end{array}\right]\left[\begin{array}{c}
-A_{\theta_{1}} H_{\theta_{1}} \\
-A_{\theta_{2}} H_{\theta_{2}} \\
-B_{1}^{c}
\end{array}\right] \tilde{y}_{\theta_{1}}(t)} \\
& {\left[\begin{array}{c}
u(t) \\
\hat{y}_{\theta_{1}}(t) \\
\hat{y}_{\theta_{2}}(t)
\end{array}\right]=\left[\begin{array}{ccc}
0 & 0 & C_{1}^{c} \\
C_{\theta_{1}} & 0 & 0 \\
0 & C_{\theta_{2}} & 0
\end{array}\right] .}
\end{aligned}
$$

Since controller $K_{1}$ stabilizes the process while the unknown parameter is $\theta_{1}$, it can be easily concluded that the injected system is stable for the frozen selection of the local controller. For simplicity of argument start by assuming that fast switching does not destroy the stability of the injected system; later in this section it will be shown how to lift this assumption. The eigenvalues of the injected system are precisely the eigenvalues of the feedback interconnection of the controller $K_{i}$ and the nominal system with $\theta_{i}$ as the value of parametric uncertainty, together with some of the (stable) eigenvalues of the other estimators and any unobservable or uncontrollable eigenvalues of the $K_{i}^{\prime}$ 's realization. The above reasoning ensures that there exist a finite constant $\gamma$ such that

$$
\left\|u^{T}(\tau) u(\tau)\right\|_{(0, t)} \leqslant \gamma\left\|\tilde{y}_{\theta_{\tilde{\sigma}_{t}(\tau)}^{T}}(\tau) \Sigma_{\theta_{\tilde{\sigma}_{t}(\tau)}}^{-1} \tilde{y}_{\tilde{\sigma}_{t}(\tau)}(\tau)\right\|_{(0, t)}+\zeta_{0}
$$

where $\zeta_{0}$ only depends on the initial condition.

Using (10) and (12), it follows that

$$
\left\|\mu_{\tilde{\sigma}_{t}(\tau)}(\tau)\right\|_{(0, t)} \leqslant N\left\|\mu_{\theta_{i}}(\tau)\right\|_{(0, t)} \leqslant N C_{0}+N C_{w}\left\|w^{T}(\tau) w(\tau)\right\|_{(0, t)}
$$

$+N C_{v}\left\|v^{T}(\tau) v(\tau)\right\|_{(0, t)}+\epsilon N C_{\epsilon}\left\|u^{T}(\tau) u(\tau)\right\|_{(0, t)}+t N \ln \beta_{\theta_{i}}$.

Combining (17) and (16),

$$
\begin{aligned}
\left\|\mu_{\tilde{\sigma}_{t}(\tau)}(\tau)\right\|_{(0, t)} \leqslant N C_{0} & +N C_{w}\left\|w^{T}(\tau) w(\tau)\right\|_{(0, t)} \\
+N C_{v}\left\|v^{T}(\tau) v(\tau)\right\|_{(0, t)} & +\epsilon \gamma N C_{\epsilon}\left\|m_{\theta_{\tilde{\sigma}_{t}(\tau)}}(\tau)\right\|_{(0, t)} \\
& +\epsilon N C_{\epsilon} \zeta_{0}+t N \ln \beta_{\theta_{i}},
\end{aligned}
$$

from which it follows that

$$
\begin{array}{r}
\left\|m_{\theta_{\tilde{\sigma}_{t}(\tau)}}(\tau)\right\|_{(0, t)} \leqslant N C_{0}+N C_{w}\left\|w^{T}(\tau) w(\tau)\right\|_{(0, t)} \\
+N C_{v}\left\|v^{T}(\tau) v(\tau)\right\|_{(0, t)}+\epsilon \gamma N C_{\epsilon}\left\|m_{\theta_{\tilde{\sigma}_{t}(\tau)}}(\tau)\right\|_{(0, t)} \\
+\epsilon N C_{\epsilon} \zeta_{0}+t N \ln \beta_{\theta_{i}}-t \ln \beta_{\theta_{\sigma(\tau)}}
\end{array}
$$

where $m_{\theta_{i}}(t)$ is introduced in (3a); it can now be concluded that

$$
\begin{aligned}
& \left\|m_{\theta_{\tilde{\sigma}_{t}(\tau)}}(\tau)\right\|_{(0, t)} \leqslant \frac{N C_{0}+\epsilon N C_{\epsilon} \zeta_{0}}{1-\epsilon \gamma N C_{\epsilon}}+\frac{t\left(\ln \beta_{\theta_{i}}^{N}-\ln \beta_{\theta_{\sigma(\tau)}}\right)}{1-\epsilon \gamma N C_{\epsilon}} \\
& +\frac{N C_{w}}{1-\epsilon \gamma N C_{\epsilon}}\left\|w^{T}(\tau) w(\tau)\right\|_{(0, t)}+\frac{N C_{v}}{1-\epsilon \gamma N C_{\epsilon}}\left\|v^{T}(\tau) v(\tau)\right\|_{(0, t),}
\end{aligned}
$$

where

$$
\epsilon<\frac{1}{\gamma N C_{\epsilon}}
$$

or equivalently,

$$
\begin{aligned}
& \frac{1}{t} \sum_{1}^{t}\left(\tilde{y}_{\theta_{\tilde{\sigma}_{t}(\tau)}}^{T}(\tau) \Sigma_{\theta_{\tilde{\sigma}_{t}(\tau)}^{-1}}^{-} \tilde{y}_{\tilde{\sigma}_{\tilde{\sigma}_{t}}(\tau)}(\tau)\right) \leqslant \frac{\bar{C}_{0}}{t}+\bar{C}_{1} \\
& +\frac{\bar{C}_{w}}{t} \sum_{1}^{t}\left(w^{T}(\tau) w(\tau)\right)+\frac{\bar{C}_{v}}{t} \sum_{1}^{t}\left(v^{T}(\tau) v(\tau)\right)
\end{aligned}
$$

where $\bar{C}_{0}=\frac{N C_{0}+\epsilon N C_{\varepsilon} \zeta_{0}}{1-\epsilon \gamma N C_{\epsilon}}, \bar{C}_{1}=\frac{\ln \beta_{\theta_{i}}^{N}-\ln \beta_{\theta_{\sigma(\tau)}}}{1-\epsilon \gamma N C_{\epsilon}}, \bar{C}_{w}=\frac{N C_{w}}{1-\epsilon \gamma N C_{\epsilon}}$, $\bar{C}_{v}=\frac{N C_{v}}{1-\epsilon \gamma N C_{\epsilon}}$, and $\epsilon<\frac{1}{\gamma N C_{\epsilon}}$.

From this bound on $\tilde{y}_{\tilde{\sigma}_{\tilde{\sigma}_{t}(\tau)}}(\tau)$, recalling that the input to the injected system is $\tilde{y}_{\tilde{\sigma}_{t}(\tau)}(\tau)$ and the injected system is exponentially stable, it is straightforward to conclude that the induced operator norm $\|.\|_{(0, t)}$ from $w$ and $v$ to any other signals and especially $u$ and $y$ is finite. Furthermore, observability of the plant will ensure that if $u$ and $y$ are bounded, then all signals remain bounded, provided that $w(t)$ and $v(t)$ are uniformly bounded for $t \in\{0,1, \ldots\}$.

The reasoning above is rigorous under the following conditions: i) there is no dwell-time switching logic and ii) the time interval between two consecutive switchings is large enough such the stability of the injected system is 
not lost due to the fast switching among stable systems. The latter is needed to avoid the stability loss of injected systems that can be caused by fast switching among the stable systems. In fact, despite the fact that the injected system is stable for a "frozen" selection of local controller, switching fast may destroy this stability. In general, when one has a linear switched system (with stable sub-systems) there is always a value for the dwell-time for which one can guarantee stability. Moreover, the computation of this minimum dwell-time can be done in several ways (see e.g., section 2.2 of Hespanha [2004]).

Using the dwell-time unit in the feedback loop, it turns out that a similar argument (to the one at the beginning of this section) can be made even if $\theta_{\tilde{\sigma}_{t}(\tau)}$ is not equal to $\operatorname{argmin}\left\{\mu_{\theta_{j}}(\tau)\right\}$, where $j=1, \ldots, N$, for $0 \leqslant \tau \leqslant t$; even though at these sampling times the injected system may not be (frozen time) stable, this may only occur over a uniformly bounded set of time with total length of no larger than $(N+1) \tau_{D}$ sampling times, which cannot destroy (exponential) stability for a linear system. In this case, an appropriate $\gamma$ can be found for which (16) is satisfied (see Hespanha [2001]).

The proposed controller was evaluated through the benchmark example of mass spring dashpot (see Fekri et al. [2006]). We used different values of the spring constant (uncertain parameter in the plant) in the simulations and the correct model is always identified. Moreover, we used the LTI local controllers that were designed by using mixed- $\mu$ synthesis methodology (see Balas [2009]), to control the uncertain plants. We applied our modified RMMAC to mass spring dashpot plant with different time varying waveform for the uncertain parameter. The identification module successfully tracked the changes in the parameter and selected the correct controller. The results are not shown due to the space limitations and can be found in Hassani et al. [2010a].

\section{CONCLUSION}

We analyzed the stability issues of robust adaptive control in which switching occurs among a bank of local controllers. We showed how a modified version of the previous architecture for Robust Adaptive Control of uncertain plants using Multiple Models (the RMMAC methodology, Fekri et al. [2006]) using the tools developed in Supervisory Control yields boundedness of all the internal signals of the resulting closed-loop system. Future work will focus on the effect of unmodelled dynamics on the proposed adaptive control solution.

\section{ACKNOWLEDGEMENTS}

We thank our colleagues A. Pedro Aguiar, Paulo Rosa for many discussions on adaptive control.

\section{REFERENCES}

B. D. O. Anderson, T. S. Brinsmead, F. De Bruyne, J. Hespanha, D. Liberzon, and A. S. Morse. Multiple model adaptive control: Part I: Finite controller coverings. Int. J. of Robust and Nonlinear Control, 10:909-929, 2000.

G. J. Balas. mixed- $\mu$ software (unpublished version). private communication, 2009.
Y. Baram and N.R. Sandell. An information theoretic approach to dynamical systems modeling and identification. IEEE Trans. on Automat. Contr., 23:61-66, 1978.

S. Fekri, M. Athans, and A. Pascoal. Issues, progress and new results in robust adaptive control. Int. J. of Adaptive Control and Signal Processing, 20:519-579, 2006.

S. Fekri, M. Athans, and A. Pascoal. Robust multiplemodel adaptive control (RMMAC): A case study. Int. J. of Adaptive Control and Signal Processing, 21:1-30, 2007.

Vahid Hassani, A. Pedro Aguiar, Antonio M. Pascoal, and Michael Athans. A performance based model-set design strategy for multiple model adaptive estimation. In ECC'09 - European Control Conference, Budapest, Hungary, 2009.

Vahid Hassani, J. Hespanha, Michael Athans, and Antonio M. Pascoal. Stability analysis of robust multiple model adaptive control. Technical Report ISR-IST-May10-01, Institute for Systems and Robotics (ISR), Instituto Superior Técnico (IST), Lisbon, Portugal, 2010a.

Vahid Hassani, Antonio M. Pascoal, A. Pedro Aguiar, and Michael Athans. A multiple model adaptive wave filter for dynamic ship positioning. In Proc. 8th IFAC Conference on Control Applications in Marine Systems (CAMS10), Rostock, Germany, 2010b.

J. Hespanha, D. Liberzon, A. S. Morse, B. D. O. Anderson, T. S. Brinsmead, and F. De Bruyne. Multiple model adaptive control: Part II: Switching. Int. J. of Robust and Nonlinear Control, 11:479-496, 2001.

J. P. Hespanha. Tutorial on supervisory control. Lecture Notes for the workshop Control using Logic and Switching for the 40th Conf. on Decision and Contr., Orlando, Florida, 2001.

J. Pedro Hespanha, D. Liberzon, and A. S. Morse. Overcoming the limitations of adaptive control by means of logic-based switching. Systems and Control Letters, 49(1): 49-65, 2003.

João Pedro Hespanha. Stabilization through hybrid control. In Encyclopedia of Life Support Systems (EOLSS), volume Control Systems, Robotics, and Automation. Developed under the Auspices of the UNESCO, Eolss Publishers, Oxford, UK, 2004.

D. G. Lainiotis. Partitioning: A unifying framework for adaptive systems I: Estimation II: Control. IEEE Trans. on Automat. Contr., 64:1182-1198, 1976.

Paulo Rosa, M. Athans, S. Fekri, and C. Silvestre. Further evaluation of the (RMMAC) method with time-varying parameters. In Proc. of 15th Mediterranean Conference on Control and Automation (MED07), Athens, Greece, 2007a.

Paulo Rosa, M. Athans, S. Fekri, and C. Silvestre. Evaluation of the (RMMAC/XI) method with time-varying parameters and disturbance statistics. In Proc. of 15th Mediterranean Conference on Control and Automation (MED07), Athens, Greece, 2007b.

M. G. Safonov and T. C. Tsao. The unfalsified control concept and learning. IEEE Trans. on Automat. Contr., 42:843-847, 1997.

G. J. Schiller and P. S. Maybeck. Control of a large space structure using MMAE/MMAC techniques. IEEE Transactions on Aerospace and Electronic System, 33:11221131, 1997.

F. L. Sims, D. G. Lainiotis, and D. T. Magill. Recursive algorithm for the calculation of the adaptive kalman filter weighting coefficient. IEEE Trans. on Automat. Contr., pages 215-218, 1969. 